\title{
Geometric properties of Bernoulli-type minimizers
}

\author{
ARshak Petrosyan ${ }^{\dagger}$ \\ Department of Mathematics, Purdue University, West Lafayette, IN 47907, USA \\ AND \\ ENRICO VALDINOCI ${ }^{\ddagger}$ \\ Dipartimento di Matematica, Università di Roma Tor Vergata, I-00133 Roma, Italy
}

[Received 2 June 2003 and in revised form 8 September 2004]

\begin{abstract}
We consider a Bernoulli-type variational problem and we prove some geometric properties for minimizers, such as: gradient bounds, linear growth from the free boundary, density estimates, uniform convergence of level sets and the existence of plane-like minimizers in periodic media.
\end{abstract}

2000 Mathematics Subject Classification: Primary 49J10, 35R35; Secondary 35J70.

Keywords: Free boundary problem; Bernoulli-type problem; $p$-Laplacian; density estimates; planelike minimizers.

\section{Introduction}

In this paper we study various geometric properties of minimizers of the energy functional

$$
\mathcal{J}_{\Omega}(u)=\int_{\Omega}\left[A(x, \nabla u)+Q(x) \chi_{(-1,1)}(u)\right] \mathrm{d} x,
$$

where $\Omega$ is a (possibly unbounded) domain in $\mathbb{R}^{n}$. We assume that $A(x, \eta), \eta \in \mathbb{R}^{n}$, behaves as $|\eta|^{p}$ for some $1<p<\infty$ and that $Q(x)>0$ is uniformly bounded away from zero and infinity (see below for precise hypotheses).

One can think of the set $\{-1<u<1\}$ as a jet of fluid in a certain medium and that $A$ and $Q$ reflect the underlying physics and geometry. In this interpretation $u$ is the stream function. In fact, the minimizers satisfy (in a distributional sense) the equation

$$
\operatorname{div} a(x, \nabla u)=0 \quad \text { in }\{-1<u<1\},
$$

where

Moreover on the free boundary

$$
a(x, \eta):=D_{\eta} A(x, \eta)
$$

$$
\Gamma:=\partial\{-1<u<1\} \cap \Omega
$$

a Bernoulli-type condition (hence the name)

$$
b(x, \nabla u)=Q(x)
$$

is satisfied in a certain weak sense (see e.g. [AC81]). Here $b(x, \eta):=\eta \cdot a(x, \eta)-A(x, \eta)$ and behaves as $|\eta|^{p}$ under the conditions we impose on $A$ and $a$.

\footnotetext{
†E-mail: arshak@math.purdue.edu
}

E-mail: valdinoci@mat.uniroma2.it 
The functional $\mathcal{J}$ and its "one-side" analogue (see Lemma 3.3 .

$$
\mathcal{F}_{\Omega}(v)=\int_{\Omega}\left[A(x, \nabla v)+Q(x) \chi_{(0, \infty)}(v)\right]
$$

have been studied earlier in the cases

$$
A(x, \eta)=|\eta|^{2}, \quad A(x, \eta)=a_{i j}(x) \eta_{i} \eta_{j}, \quad A(x, \eta)=|\eta|^{p}
$$

respectively by [AC81], [Val01] and [DP03] and we borrow many of the ideas from those papers. We prove gradient bounds, linear growth from the free boundary, density estimates, uniform convergence of level sets to a set of minimal "perimeter" (see [Bou90]) and the existence of planelike minimizers in periodic media. For the latter two results, we were influenced mostly by [CC95] and [CdlL01].

We now state explicitly the hypotheses we assume on our functional:

(H1) $\quad A \in C^{1}\left(\mathbb{R}^{n} \times \mathbb{R}^{n}\right), a \in C\left(\mathbb{R}^{n} \times \mathbb{R}^{n}\right) \cap C^{1}\left(\mathbb{R}^{n} \times \mathbb{R}^{n}-\{0\}\right)$;

$$
A(x, 0)=0, a(x, 0)=0
$$

(H2) $\zeta \cdot D_{\eta} a(x, \eta) \zeta \geqslant \lambda|\zeta|^{2}|\eta|^{p-2}$ for any $\zeta \in \mathbb{R}^{n}$;

in particular, $A(x, \eta)$ is convex in $\eta$;

(H3) $\quad\left|D_{\eta} a(x, \eta)\right| \leqslant \Lambda|\eta|^{p-2}$;

(H4) $\quad\left|D_{x} a(x, \eta)\right| \leqslant \Lambda|\eta|^{p-1}$;

(H5) $\quad|a(x, \eta)| \leqslant \Lambda|\eta|^{p-1}$;

(H6) $\quad \eta \cdot a(x, \eta) \geqslant \lambda|\eta|^{p}$;

(H7) $0<Q_{\min } \leqslant Q(x) \leqslant Q_{\max }$.

Here and in what follows we assume $\lambda \leqslant \Lambda$ and $Q_{\min } \leqslant Q_{\max }$ to be strictly positive constants. We will refer to $\lambda, \Lambda, Q_{\min }, Q_{\max }, p$ and $n$ as the structural constants. Quantities depending only on them will be referred as universal constants. Hypotheses (H1)-(H5) are needed in order to apply the interior regularity results of [Tol84]. Under hypothesis (H6), we are also able to employ the Harnack inequality of [Tru67].

Also, when dealing with global geometric results, we will consider periodic media, i.e. we will require the following condition:

(H8) $\quad A(x, \eta)=A(x+k, \eta)$ and $Q(x)=Q(x+k)$ for any $k \in \mathbb{Z}^{n}$.

In what follows, we will take $u \in L_{\text {loc }}^{1}$ with $\nabla u \in L_{\text {loc }}^{p}$ and we will consider several types of minimizers for the functional $\mathcal{J}$. Namely, if $K \subset \mathbb{R}^{n}$ is a compact domain, we say that $u$ is an absolute minimizer for $\mathcal{J}$ in $K$ if $\mathcal{J}_{K}(u) \leqslant \mathcal{J}_{K}(u+\varphi)$ for any $\varphi \in C_{0}^{\infty}(K)$.

We say that a function $u$ in $\mathbb{R}^{n}$ is a class $A$ minimizer if $\mathcal{J}_{K}(u) \leqslant \mathcal{J}_{K}(u+\varphi)$ for any compact domain $K \subset \mathbb{R}^{n}$ and for any $\varphi \in C_{0}^{\infty}(K)$; i.e., a class $A$ minimizer is an absolute minimizer in any compact domain.

Also, given a domain $\Omega$ in $\mathbb{R}^{n}$, a subset $\Sigma \subset \partial \Omega$ and a function $-1 \leqslant u_{0} \leqslant 1$ we consider the class $\mathcal{X}=\mathcal{X}_{\Omega, \Sigma, u_{0}}$ of functions that are equal to $u_{0}$ on $\Sigma$ (see 3.1 below). We say that $u$ is a constrained minimizer if $\mathcal{J}_{\Omega}(u) \leqslant \mathcal{J}_{\Omega}(v)$ for any $v \in \mathcal{X}$.

\section{Main results}

Below we state the main results of this paper.

As a first result, we prove uniform gradient bounds and linear growth from the free boundary for minimizers of 1.3 . 
THEOREM 2.1 Assume hypotheses (H1)-(H7). Let $v$ be a constrained minimizer for $\mathcal{F}$ in a domain $\Omega$. Then $v$ is Lipschitz continuous. Moreover, given $K \subset \subset \Omega$, the Lipschitz norm of $v$ on $K$ depends only on the structural constants and the distance between $K$ and $\partial \Omega$. Also, if $B_{r}(x) \subset K \cap\{v>0\}$ touches $\partial\{v>0\}$, then

$$
c r \leqslant v(x) \leqslant C r
$$

for suitable positive universal constants $c$ and $C$.

This result is an extension of [AC81], [Val01] and [DP03], which have considered, respectively, the cases $A(x, \eta)=|\eta|^{2}, A(x, \eta)=a_{i j}(x) \eta_{i} \eta_{j}$ and $A(x, \eta)=|\eta|^{p}$.

Next, we prove the following density estimates for the level sets of minimizers:

THEOREM 2.2 Assume hypotheses (H1)-(H7). Let $u$ be a constrained minimizer for $\mathcal{J}$ in a domain $\Omega$ and $x \in\{-1<u<1\}$. Then, for fixed $r_{1}>0$, there exist positive $r_{0}, c$ and $C$ depending only on the structural constants and $r_{1}$ such that

$$
\begin{gathered}
c r^{n-1} \leqslant \mathcal{J}_{B_{r}(x)}(u) \leqslant C r^{n-1}, \\
\mathcal{L}^{n}\left(B_{r}(x) \cap\{u=-1\}\right) \geqslant c r^{n} \quad \text { and } \quad \mathcal{L}^{n}\left(B_{r}(x) \cap\{u=1\}\right) \geqslant c r^{n},
\end{gathered}
$$

for any $r \geqslant r_{0}$ provided $B_{r+r_{1}}(x) \subset \subset \Omega$.

Analogous density estimates for $A(x, \eta)=|\eta|^{2}$ and $A(x, \eta)=a_{i j}(x) \eta_{i} \eta_{j}$ have been dealt with in [CC95] and [Val01], respectively.

By $\Gamma$-convergence methods, it has been proved in [Bou90] that minimizers $u_{\varepsilon}$ of

$$
\mathcal{J}^{\varepsilon}(u)=\int\left[A(x, \varepsilon \nabla u)+Q(x) \chi_{(-1,1)}(u)\right]
$$

converge in $L_{\text {loc }}^{1}$ to a function $u_{0}$ which has a minimal "interface" with respect to some weighted area. Indeed, a consequence of the above density estimates is that level sets converge in $L_{\text {loc }}^{\infty}$ :

THEOREM 2.3 Assume hypotheses (H1)-(H7). Let $u_{\varepsilon}$ be an absolute minimizer of (2.1) in a bounded domain $D$. Assume that, as $\varepsilon \rightarrow 0, u_{\varepsilon}$ converges in $L_{\text {loc }}^{1}$ to

$$
u_{0}:=\chi_{E}-\chi_{D-E}
$$

for a suitable $E \subset D$. Then $\left\{\left|u_{\varepsilon}\right|<1\right\}$ converges locally uniformly to $\partial E$.

The latter convergence is understood in the sense that $\operatorname{dist}(x, \partial E) \rightarrow 0$ uniformly for $x \in$ $\left\{\left|u_{\varepsilon}\right|<1\right\} \cap K$ for any $K \subset \subset D$.

Finally, we study the minimizers of $\mathcal{J}$ in periodic media. We prove the existence of class $A$ minimizers in periodic media constrained in a strip of universal width. Also, we show that any periodic minimizer constrained in a strip wide enough is indeed unconstrained and class $A$. More precisely, we prove:

THEOREM 2.4 Assume hypotheses (H1)-(H8). Then there exists a positive universal constant $M_{0}$ such that:

(T1) Given any $\omega \in \mathbb{R}^{n}-\{0\}$, there exists a class $A$ minimizer $u=u_{\omega}$ for the functional $\mathcal{J}$ for which the set $\{|u|<1\}$ is constrained in the strip $\left\{x \cdot \omega \in\left[0, M_{0}|\omega|\right]\right\}$.

Furthermore, such $u$ enjoys the following property of "quasi-periodicity": 
- if $\omega \in \mathbb{Q}^{n}-\{0\}$, then $u$ is periodic (with respect to the identification induced by $\omega$ );

- if $\omega \in \mathbb{R}^{n}-\mathbb{Q}^{n}$, then $u$ can be approximated uniformly on compact sets by periodic class $A$ minimizers.

(T2) If $\omega \in \mathbb{Q}^{n}-\{0\}$ and $v$ is any $\omega$-periodic minimizer of $\mathcal{J}$ constrained in the strip $S_{M}^{\omega}:=$ $\{x \cdot \omega \in[0, M|\omega|]\}, M \geqslant M_{0}$, then it is also an unconstrained class $A$ minimizer.

Notice that $M_{0}$ above is independent of the frequency $\omega$.

The paper is organized as follows. In Section 3, we discuss existence of constrained minimizers and in Section 4 we prove optimal Lipschitz regularity (i.e., the first part of Theorem 2.1). Section 5 deals with the linear growth from the free boundary (i.e., the second part of Theorem 2.1). The density estimates and the uniform convergence of level sets will be dealt with in Section 6 , where we also prove Theorems 2.2 and 2.3 In Section 7 we recall a geometric result, which will be of use in Section 8, where the proof of Theorem 2.4 will be carried out. The Appendix collects some utilities. The results in Sections 377 will make use only of hypotheses (H1)-(H7), while in Section 8 we will also make use of hypothesis (H8).

\section{Existence}

In this section we show the existence and recall some properties of the constrained minimizers of the functional

$$
\mathcal{J}_{\Omega}(u)=\int_{\Omega}\left[A(x, \nabla u)+Q(x) \chi_{(-1,1)}(u)\right]
$$

over the class of admissible functions

$$
\mathcal{X}_{\Omega}=\mathcal{X}_{\Omega, \Sigma, u_{0}}:=\left\{u \in L_{\mathrm{loc}}^{1}(\Omega): \nabla u \in L^{p}(\Omega) \text { and } u=u_{0} \text { on } \Sigma\right\} .
$$

Here $\Omega$ is a (possibly unbounded) domain in $\mathbb{R}^{n}$ with Lipschitz regular boundary $\partial \Omega, \Sigma$ is a given measurable subset of $\partial \Omega$ with $\mathcal{H}^{n-1}(\Sigma)>0$ and $u_{0}$ is a given function with

$$
-1 \leqslant u_{0} \leqslant 1, \quad u_{0} \in L_{\mathrm{loc}}^{1}(\Omega), \quad \nabla u_{0} \in L^{p}(\Omega) .
$$

We start by discussing the known existence and regularity of such constrained minimizers.

Proposition 3.1 (i) Suppose that $\mathcal{J}_{\Omega}\left(u_{1}\right)<\infty$ for some $u_{1} \in \mathcal{X}_{\Omega}$. Then there exists a constrained minimizer of $\mathcal{J}_{\Omega}$ in the class $\mathcal{X}_{\Omega}$.

(ii) Every constrained minimizer $u$ is Hölder continuous in the interior of $\Omega$. Furthermore, given $K \subset \subset \Omega$, the Hölder exponent $0<\alpha<1$ and the $C^{\alpha}$ norm of $u$ on $K$ depend only on the structural constants and the distance between $K$ and $\partial \Omega$.

Proof. (i) The existence is obtained by a direct method of Calculus of Variations. Our proof below is the same as in [AC81, Proposition 1.3] (see also [Val01, Theorem 9.1]).

Let $u_{k}$ be a minimizing sequence for $\mathcal{J}_{\Omega}$ in the sense that

$$
\mathcal{J}_{\Omega}\left(u_{k}\right) \rightarrow \inf _{\mathcal{X}_{\Omega}} \mathcal{J}_{\Omega}
$$

Then from (H1), (H2) and (H6) it follows that

$$
A(x, \eta) \geqslant \lambda|\eta|^{p},
$$


hence the sequence $\nabla u_{k}$ is uniformly bounded in $L^{p}(\Omega)$. Moreover, $u_{k}-u_{0}$ is uniformly bounded in $L^{p}\left(\Omega \cap B_{R}\right)$ (since $\mathcal{H}^{n-1}(\Sigma)>0$ ) for any large $R$. Thus we can extract a subsequence (still denoted by $u_{k}$ ) such that

$$
\begin{aligned}
\nabla u_{k} \rightarrow \nabla u & \text { weakly in } L^{p}(\Omega), \\
u_{k} \rightarrow u & \text { a.e. in } \Omega .
\end{aligned}
$$

Since we assume $A(x, \eta)$ is convex in $\eta$ we have (see, for instance, Theorem 1.2 in [Dac89])

$$
\int_{\Omega \cap B_{R}} A(x, \nabla u) \leqslant \liminf _{k \rightarrow \infty} \int_{\Omega \cap B_{R}} A\left(x, \nabla u_{k}\right) .
$$

Moreover, the pointwise convergence implies

$$
\int_{\Omega \cap B_{R}} Q(x) \chi_{(-1,1)}(u) \leqslant \liminf _{k \rightarrow \infty} \int_{\Omega \cap B_{R}} Q(x) \chi_{(-1,1)}\left(u_{k}\right),
$$

since $\chi_{(-1,1)}$ is lower semicontinuous, and letting $R \rightarrow \infty$ we obtain

$$
\mathcal{J}_{\Omega}(u) \leqslant \liminf _{k \rightarrow \infty} \mathcal{J}_{\Omega}\left(u_{k}\right) .
$$

(ii) The Hölder regularity of minimizers follows from a theorem of Giaquinta and Giusti [GG82].

Since the functional $\mathcal{J}_{\Omega}$ is not convex, there could be more than one minimizer. We will denote by $\mathcal{M}_{\Omega}$ the class of all constrained minimizers in $\mathcal{X}_{\Omega}$. Observe that for any $u \in \mathcal{M}_{\Omega}$,

$$
-1 \leqslant u \leqslant 1 \quad \text { a.e. in } \Omega .
$$

Indeed, otherwise $\max (\min (u, 1),-1)$ would have less energy.

Now we turn to the PDE properties of minimizers.

Proposition 3.2 Let $u \in \mathcal{M}_{\Omega}$. Then in the weak (distributional) sense

$$
\operatorname{div} a(x, \nabla u)=0 \quad \text { in }\{-1<u<1\} .
$$

Moreover, on the free boundary $\Gamma=\partial\{-1<u<1\} \cap \Omega$ a Bernoulli-type condition

$$
b(x, \nabla u)=Q(x)
$$

is satisfied in a certain weak sense, where $b(x, \eta):=a(x, \eta) \cdot \eta-A(x, \eta)$.

Observe that the structural conditions imply that $c|\eta|^{p} \leqslant b(x, \eta) \leqslant C|\eta|^{p}$.

Proof. We leave the standard proof of (3.2) to the reader. For the interpretation of the condition (3.3) we refer to [AC81].

The free boundary $\Gamma$ in the proposition above consists of two parts

$$
\Gamma_{i}=\partial\{-1<u<1\} \cap\{u=i\}, \quad i=-1,1,
$$

that are well separated on every compact subset of $\Omega$, by Hölder regularity of $u$. The following simple observation will be extensively used throughout the paper. 
LEMMA 3.3 Suppose that in a subdomain $D \subset \subset \Omega$ the minimizer $u$ does not take the value 1 (respectively -1 ). Then the function $v=u+1$ (respectively $v=1-u$ ) is an absolute minimizer of the functional

$$
\mathcal{F}_{D}(v)=\int_{D}\left[A(x, \nabla v)+Q(x) \chi_{(0, \infty)}(v)\right]
$$

We conclude this section with a remark about rescalings.

We will use two kinds of rescalings. The first kind preserves the gradient and is suitable for proving the Lipschitz regularity of minimizers. It is better formulated in terms of the functional $\mathcal{F}$. Namely, let $v$ be a minimizer of $\mathcal{F}_{\Omega}$ and $x_{0} \in \mathbb{R}^{n}$. Then the function

$$
v_{\varepsilon}(x)=\frac{1}{\varepsilon} v\left(x_{0}+\varepsilon x\right)
$$

is a minimizer of

$$
\int_{\Omega_{1 / \varepsilon}}\left[A_{\varepsilon}(x, \nabla v)+Q_{\varepsilon}(x) \chi_{(0, \infty)}(v)\right],
$$

where $\Omega_{1 / \varepsilon}=\Omega_{x_{0}, 1 / \varepsilon}=\left\{x: x_{0}+\varepsilon x \in \Omega\right\}$ and

$$
A_{\varepsilon}(x, \eta)=A\left(x_{0}+\varepsilon x, \eta\right), \quad Q_{\varepsilon}(x)=Q\left(x_{0}+\varepsilon x\right) .
$$

Note that $A_{\varepsilon}$ and $Q_{\varepsilon}$ satisfy the same (if not better) structural conditions as $A$ and $Q$.

The second kind of rescalings preserves the value of the function. We will need it in the proof of Theorem 2.3 (see the end of Section 6). We state it in terms of the functional $\mathcal{J}$. Namely, if $u_{\varepsilon}$ is a minimizer of

Then the function

$$
\int_{\Omega}\left[A(x, \varepsilon \nabla u)+Q(x) \chi_{(-1,1)}(u)\right]
$$

$$
w_{\varepsilon}(x)=u_{\varepsilon}\left(x_{0}+\varepsilon x\right)
$$

is a minimizer of

$$
\int_{\Omega_{1 / \varepsilon}}\left[A_{\varepsilon}(x, \nabla w)+Q_{\varepsilon}(x) \chi_{(-1,1)}(w)\right],
$$

with $A_{\varepsilon}$ and $Q_{\varepsilon}$ as in 3.5 .

\section{Lipschitz regularity}

In this section we prove that every constrained minimizer $u$ of $\mathcal{J}$ is in fact Lipschitz.

THEOREM 4.1 Let $u \in \mathcal{M}_{\Omega}$. Then $u$ is Lipschitz continuous. Moreover, given $K \subset \subset \Omega$, the Lipschitz norm of $u$ on $K$ depends only on the structural constants and the distance between $K$ and $\partial \Omega$.

The idea is to show that $u$ grows at most linearly away from the free boundary

$$
\Gamma=\partial\{-1<u<1\} .
$$

The Hölder regularity of $u$ implies that the two parts of the free boundary, $\Gamma_{ \pm 1}$, are well separated on $K$ in the sense that

$$
\operatorname{dist}\left(\Gamma_{-1} \cap K, \Gamma_{1} \cap K\right) \geqslant \delta_{0}>0 .
$$


Let now $x_{0} \in \Gamma \cap K$. Without loss of generality we may assume that $x_{0} \in \Gamma_{-1} \cap K$. Then for $\varepsilon<\min \left(\delta_{0}, \operatorname{dist}(K, \partial \Omega)\right)$, the ball $B_{\varepsilon}\left(x_{0}\right)$ is contained in $\Omega$ and does not intersect $\Gamma_{1}$. Hence the function $v=u+1$ will be a minimizer of $\mathcal{F}_{B_{\varepsilon}\left(x_{0}\right)}$, as defined in Lemma 3.3

The next lemma is the main step in establishing Theorem 4.1. The proof is based on that of Lemma 3.2 in [DP03].

Lemma 4.2 Let $v$ be a bounded absolute minimizer of $\mathcal{F}_{B_{\varepsilon}\left(x_{0}\right)}$ with $v\left(x_{0}\right)=0$. Then

$$
\sup _{B_{\varepsilon / 4}\left(x_{0}\right)} v \leqslant C \varepsilon
$$

and

$$
\left|\nabla v\left(x_{0}\right)\right| \leqslant C,
$$

where $C>0$ is a universal constant.

Proof. Observe that 4.2 is a consequence of 4.1 and the interior gradient estimates (see e.g. [Tol84]). Moreover, by the rescaling arguments at the end of the previous section, it is enough to consider the case $\varepsilon=1$ and $x_{0}=0$ to prove 4.1 .

Next we argue by contradiction. Indeed, assume that the estimate (4.1) is false for $\varepsilon=1$. Then there exists a sequence of bounded absolute minimizers $v_{k}$ of the functionals

$$
\mathcal{F}^{k}(v)=\int\left[A^{k}(x, \nabla v)+Q^{k}(x) \chi_{(0, \infty)}(v)\right]
$$

in $B_{1}, k=1,2, \ldots$, such that

$$
\max _{\bar{B}_{1 / 4}} v_{k}(x)>k .
$$

Set

$$
d_{k}(x)=\operatorname{dist}\left(x,\left\{v_{k}=0\right\}\right) \quad \text { in } B_{1}
$$

and define

$$
\mathcal{O}_{k}=\left\{x \in B_{1}: d_{k}(x) \leqslant(1-|x|) / 3\right\} .
$$

Observe that $\bar{B}_{1 / 4} \subset \mathcal{O}_{k}$. In particular

$$
m_{k}:=\sup _{\mathcal{O}_{k}}(1-|x|) v_{k}(x) \geqslant \frac{3}{4} \max _{\bar{B}_{1 / 4}} v_{k}(x)>\frac{3}{4} k .
$$

Since $v_{k}$ is bounded (for fixed $k$ ), we have $(1-|x|) v_{k}(x) \rightarrow 0$ as $|x| \rightarrow 1$, and therefore $m_{k}$ will be attained at some point $x_{k} \in \mathcal{O}_{k}$ :

$$
\left(1-\left|x_{k}\right|\right) v_{k}\left(x_{k}\right)=\max _{\mathcal{O}_{k}}(1-|x|) v_{k}(x) .
$$

Clearly,

$$
v_{k}\left(x_{k}\right)=\frac{m_{k}}{1-\left|x_{k}\right|} \geqslant m_{k}>\frac{3}{4} k .
$$

Since $x_{k} \in \mathcal{O}_{k}$, by the definition we have

$$
d_{k}:=d_{k}\left(x_{k}\right) \leqslant\left(1-\left|x_{k}\right|\right) / 3 .
$$


Let now $y_{k} \in \partial\left\{v_{k}>0\right\} \cap B_{1}$ be such that

$$
\left|y_{k}-x_{k}\right|=d_{k} .
$$

Then (4.4)-(4.5) imply that

$$
B_{2 d_{k}}\left(y_{k}\right) \subset B_{1} \quad \text { and } \quad B_{d_{k} / 2}\left(y_{k}\right) \subset \mathcal{O}_{k} .
$$

In particular, for $z \in B_{d_{k} / 2}\left(y_{k}\right)$,

$$
1-|z| \geqslant\left(1-\left|x_{k}\right|\right)-\left|x_{k}-z\right| \geqslant\left(1-\left|x_{k}\right|\right)-(3 / 2) d_{k} \geqslant\left(1-\left|x_{k}\right|\right) / 2 .
$$

This, in conjunction with 4.3 , implies that

$$
\max _{\bar{B}_{d_{k} / 2\left(y_{k}\right)}} v_{k} \leqslant 2 v_{k}\left(x_{k}\right)
$$

Next, since $B_{d_{k}}\left(x_{k}\right) \subset\left\{v_{k}>0\right\}, v_{k}$ satisfies

$$
\operatorname{div} a^{k}\left(x, \nabla v_{k}\right)=0 \quad \text { in } B_{d_{k}}\left(x_{k}\right)
$$

with $a^{k}=D_{\eta} A^{k}$. By the Harnack inequality (see Theorem 1.1-1.3 in [Tru67]), there is a constant $c>0$ such that

$$
\min _{\bar{B}_{3 d_{k} / 4}\left(x_{k}\right)} v_{k} \geqslant c v_{k}\left(x_{k}\right) .
$$

In particular,

$$
\max _{\bar{B}_{d_{k} / 4}\left(y_{k}\right)} v_{k} \geqslant c v_{k}\left(x_{k}\right) .
$$

Consider now the rescalings

$$
w_{k}(x)=\frac{v_{k}\left(y_{k}+\left(d_{k} / 2\right) x\right)}{v_{k}\left(x_{k}\right)} .
$$

From the properties of $v_{k}$ above, we obtain

$$
\max _{\bar{B}_{1}} w_{k} \leqslant 2, \quad \max _{\bar{B}_{1 / 2}} w_{k} \geqslant c>0, \quad w_{k}(0)=0 .
$$

Moreover, $w_{k}$ is an absolute minimizer of

$$
\tilde{\mathcal{F}}^{k}(w)=\int\left[\tilde{A}^{k}(x, \nabla w)+\tilde{Q}^{k}(x) \chi_{(0, \infty)}(w)\right]
$$

in $B_{1}$ with

$$
\begin{aligned}
\tilde{A}^{k}(x, \eta) & =\varepsilon_{k}^{p} A^{k}\left(y_{k}+\left(d_{k} / 2\right) x, \eta / \varepsilon_{k}\right), \\
\tilde{Q}^{k}(x, \mu) & =\varepsilon_{k}^{p} Q^{k}\left(y_{k}+\left(d_{k} / 2\right) x\right), \quad \varepsilon_{k}=d_{k} /\left(2 v_{k}\left(x_{k}\right)\right) .
\end{aligned}
$$

Then over a subsequence

$$
\tilde{a}^{k}(x, \eta) \rightarrow a^{0}(\eta), \quad x \in B_{1},
$$

uniformly for $\eta$ in compact subsets of $\mathbb{R}^{n}$, where $a^{k}=D_{\eta} A^{k}$ and $a^{0}$ satisfies the same structural conditions as $a^{k}$. Moreover, we have

$$
0 \leqslant Q^{k}(x) \leqslant Q_{\max } \varepsilon_{k}^{p} \rightarrow 0 .
$$


Let now $v_{k}$ be such that $v_{k}-w_{k} \in W_{0}^{1, p}\left(B_{3 / 4}\right)$ and $\operatorname{div} a^{k}\left(x, \nabla v_{k}\right)=0$ in $B_{3 / 4}$, where $a^{k}(x, \eta)=$ $D_{h} A^{k}(x, \eta)$. Then from Lemma A.1 in the Appendix we have

$$
\left\|\nabla\left(w_{k}-v_{k}\right)\right\|_{L^{p}\left(B_{3 / 4}\right)} \leqslant C\left(\varepsilon_{k}\right) \rightarrow 0 .
$$

Moreover, from a theorem of Giaquinta and Giusti [GG82], $w_{k}$ and $v_{k}$ are uniformly $C^{\alpha}$ in $B_{5 / 8}$. Thus, we can extract subsequences (still denoted by $w_{k}$ and $v_{k}$ ) such that $w_{k} \rightarrow w_{0}$ and $v_{k} \rightarrow v_{0}$ uniformly on $B_{5 / 8}$. On the other hand, $v_{k}$ satisfy uniform $C^{1, \alpha}$ estimates on $B_{5 / 8}$ (see [Tol84]). Hence, we can assume that the convergence $v_{k} \rightarrow v_{0}$ is actually in $C^{1, \beta}$ on $B_{5 / 8}$ and $v_{0}$ solves

$$
\operatorname{div} a^{0}\left(\nabla v_{0}\right)=0 \quad \text { in } B_{5 / 8} .
$$

We next observe that 4.7 implies that $w_{0}=v_{0}+c$ in $B_{5 / 8}$. Hence, also

$$
\operatorname{div} a^{0}\left(\nabla w_{0}\right)=0 \quad \text { in } B_{5 / 8} .
$$

Since $w_{0} \geqslant 0$ and $w_{0}(0)=0$, from the Harnack inequality (see [Tru67]) we deduce that $w_{0}=0$ in $B_{5 / 8}$. On the other hand, 44.6 implies

$$
\max _{\bar{B}_{1 / 2}} w_{0} \geqslant c>0,
$$

which is a contradiction.

The lemma is proved.

Proof of Theorem 4.1. Lemma 4.2 implies that $|\nabla u|$ is bounded on $\Gamma \cap K$ and we need to prove the boundedness also in $\{-1<u<1\} \cap K$. So, fix a point $x_{0} \in K$ with $-1<u\left(x_{0}\right)<1$ and consider the following two cases:

1. $\operatorname{dist}\left(x_{0}, \Gamma\right)>\delta / 2$,

2. $\operatorname{dist}\left(x_{0}, \Gamma\right) \leqslant \delta / 2$,

where

$$
\delta=\operatorname{dist}(K, \partial \Omega)>0 .
$$

In the former case we have $\left|\nabla u\left(x_{0}\right)\right| \leqslant C / \delta$ by the interior gradient estimates (see [Tol84]), and in the latter case we argue as follows. Consider the ball $B_{\varepsilon}\left(x_{0}\right)$ with $\varepsilon=\operatorname{dist}\left(x_{0}, \Gamma\right)$ and let $y_{0} \in$ $\Gamma \cap \partial B_{\varepsilon}\left(x_{0}\right)$. Then $\operatorname{dist}\left(y_{0}, \partial \Omega\right) \geqslant \delta / 2$ and by Lemma 4.2 we have $\left|\nabla u\left(y_{0}\right)\right| \leqslant C$. Without loss of generality we may also assume that $y_{0} \in \Gamma_{-1}$. We claim then

$$
c_{0}:=\inf _{B_{\kappa \varepsilon}\left(x_{0}\right)}(u+1) \leqslant C \varepsilon,
$$

where the constant $0<\kappa<1$ will be specified below and will depend only on the structural constants of $A$. Define

$$
\psi_{\kappa}(s)=s+C_{\kappa} s^{2}, \quad C_{\kappa}=\kappa /(1-\kappa)^{2} .
$$

Then $\psi_{\kappa}$ has the following properties:

$$
\psi_{\kappa}(0)=0, \quad \psi_{\kappa}(1-\kappa)=1, \quad \psi_{\kappa}(0)=1, \quad \psi_{k}^{\prime \prime}(s)=2 C_{\kappa} .
$$

Consider now the function

$$
\phi(x)=c_{0} \psi_{\kappa}\left(1-\left|x-x_{0}\right| / \varepsilon\right) \quad \text { in } U_{\kappa}:=B_{\varepsilon}\left(x_{0}\right) \backslash B_{\kappa \varepsilon}\left(x_{0}\right) .
$$


Then, using the strict convexity of $\psi_{\kappa},(\mathbf{H} 2)$ and (H4), we obtain

$$
\operatorname{div} a(x, \nabla \phi) \geqslant 0 \quad \text { in } U_{\kappa}
$$

for $\kappa_{0}<\kappa<1$ depending only on the structural constants. Moreover, $\phi \leqslant u+1$ on $\partial U_{\kappa}$, hence by the standard comparison principle $\phi \leqslant u+1$ in $U_{\kappa}$. Since also $\phi\left(y_{0}\right)=u\left(y_{0}\right)+1=0$ we obtain

$$
\left|\nabla \phi\left(x_{0}\right)\right| \leqslant\left|\nabla u\left(y_{0}\right)\right|
$$

hence

and consequently

$$
c_{0} \psi_{\kappa}^{\prime}(0) / \varepsilon \leqslant C
$$

$$
c_{0} \leqslant C \varepsilon
$$

Finally, we observe that by the Harnack inequality (see [Tru67]) we also have

$$
\sup _{B_{\kappa \varepsilon}\left(x_{0}\right)}(u+1) \leqslant C \inf _{B_{\kappa \varepsilon}\left(x_{0}\right)}(u+1) \leqslant C \varepsilon .
$$

Then applying the interior gradient estimate, we conclude the proof of the theorem.

\section{Nondegeneracy}

As we have seen in the previous section, a minimizer $u \in \mathcal{M}_{\Omega}$ grows at most linearly near the free boundary $\Gamma=\partial\{-1<u<1\}$. In this section we show that the linear growth is the optimal one.

In order to study the growth of $u$ near $\Gamma_{-1}$ (or $\Gamma_{1}$ ) we fix a domain $D \subset \subset \Omega$ in which $u$ does not take value 1 (respectively -1 ). Then the function $v=u+1$ (respectively $v=1-u$ ) is an absolute minimizer of the functional $\mathcal{F}_{D}$.

As a simple corollary from Theorem 4.1 we obtain the following statement.

LEMMA 5.1 There exists a constant $C$ depending only on the structural constants and $\operatorname{dist}(D, \partial \Omega)$ such that for any absolute minimizer $v$ of $\mathcal{F}_{D}$,

$$
\frac{1}{r} f_{\partial B_{r}} v>C \text { implies } v>0 \text { in } B_{r}
$$

for any ball $B_{r} \subset D$.

Proof. Indeed, otherwise $B_{r} \subset D$ contains a free boundary point and then, by Theorem $4.1, u \leqslant C r$ on $\partial B_{r}$, a contradiction.

Next we prove a key nondegeneracy lemma.

LEMMA 5.2 For any $\gamma>p-1$ and $0<\kappa<1$ there exists $c>0$ depending only on $\kappa, \gamma$ and the structural constants such that for every absolute minimizer $v$ of $\mathcal{F}_{D}$ and for any ball $B_{r} \subset D$,

$$
\frac{1}{r}\left(f_{B_{r}} v^{\gamma}\right)^{1 / \gamma}<c \text { implies } v=0 \text { in } B_{\kappa} r .
$$

In particular, if $B_{r} \subset D$ and $B_{\kappa r} \cap \partial\{v>0\} \neq \emptyset$, then

$$
\sup v \geqslant c r \text {. }
$$


Proof. Without loss of generality we may consider the case $r=1$. Set

$$
\varepsilon=\sup _{B_{\sqrt{\kappa}}} v
$$

By the weak Harnack inequality for subsolutions (see Theorem 1.3 in [Tru67]), we have

$$
\varepsilon \leqslant C\left(f_{B_{1}} v^{\gamma}\right)^{1 / \gamma} .
$$

Hence, if $c$ is small, so is $\varepsilon$. Let $\phi(x)=\phi_{K}(x)$ be the solution of

$$
\operatorname{div} a(x, \nabla \phi)=0 \quad \text { in } B_{\sqrt{\kappa}}-B_{\kappa}, \quad \phi=0 \quad \text { on } \partial B_{\kappa}, \quad \phi=1 \quad \text { on } \partial B_{\sqrt{\kappa}}
$$

and put $\phi=0$ on $B_{\kappa}$. Set $h=\varepsilon \phi$ in $B_{\sqrt{\kappa}}$. Then $h \geqslant v$ on $\partial B_{\sqrt{\kappa}}$ and $v^{*}=\min (v, h)$ is an admissible function. Therefore $\mathcal{F}(v) \leqslant \mathcal{F}\left(v^{*}\right)$, or, equivalently,

$$
\int_{B_{\sqrt{\kappa}}}\left[A(x, \nabla v)+Q(x) \chi_{(0, \infty)}(v)\right] \leqslant \int_{B_{\sqrt{\kappa}}-B_{\kappa}}\left[A\left(x, \nabla v^{*}\right)+Q(x) \chi_{(0, \infty)}\left(v^{*}\right)\right] .
$$

Hence

$$
\begin{aligned}
\int_{B_{\kappa}}\left[A(x, \nabla v)+Q(x) \chi_{(0, \infty)}(v)\right] & \leqslant \int_{B_{\sqrt{k}}-B_{\kappa}}\left[A\left(x, \nabla v^{*}\right)-A(x, \nabla v)\right] \\
& \leqslant \int_{B_{\sqrt{k}}-B_{\kappa}} a\left(x, \nabla v^{*}\right) \cdot \nabla\left(v^{*}-v\right) \\
& =\int_{\left(B_{\sqrt{k}}-B_{\kappa}\right) \cap\left\{v>v^{*}\right\}} a\left(x, \nabla v^{*}\right) \cdot \nabla\left(v^{*}-v\right) \\
& =-\int_{\partial B_{k}}\left(v^{*}-v\right) a\left(x, \nabla v^{*}\right) \cdot v \\
& =\int_{\partial B_{\kappa}} v a(x, \nabla h) \cdot v .
\end{aligned}
$$

Here in the second step we have used the convexity of $A(x, \eta)$ in $\eta$ (see (H2)). Since also $|\nabla h| \leqslant C \varepsilon$ on $\partial B_{\kappa}$ we find that

$$
\int_{B_{\kappa}}\left[A(x, \nabla v)+Q(x) \chi_{(0, \infty)}(v)\right] \leqslant C \varepsilon^{p-1} \int_{\partial B_{\kappa}} v .
$$

On the other hand, using the Fundamental Theorem of Calculus, polar coordinates and the Young inequality,

$$
\begin{aligned}
\int_{\partial B_{\kappa}} v & \leqslant C\left(\int_{B_{\kappa}} v+\int_{B_{\kappa}}|\nabla v|\right) \\
& \leqslant C\left(\int_{B_{\kappa}} \varepsilon Q(x) \chi_{(0, \infty)}(v)+\int_{B_{\kappa}}\left[A(x, \nabla v)+Q(x) \chi_{(0, \infty)}(v)\right]\right) \\
& \leqslant C(1+\varepsilon) \int_{B_{\kappa}}\left[A(x, \nabla v)+Q(x) \chi_{(0, \infty)}(v)\right] .
\end{aligned}
$$

Therefore, if $\varepsilon$ is small enough, we obtain $v=0$ in $B_{\kappa}$. 
In light of the result above and Theorem 4.1, we infer the following:

COROLlaRY 5.3 There exist constants $c, C>0$ such that if $B_{r}(x) \subset\{v>0\} \cap D$ touches $\partial\{v>0\} \cap D$ then

$$
c r \leqslant v(x) \leqslant C r .
$$

Theorem 2.1 follows now from this and Theorem 4.1 .

\section{Density estimates}

The purpose of this section is to show that the jet $\{-1<u<1\}$ has a bounded width in the sense that $B_{r} \cap\{-1<u<1\}$ has a Lebesgue measure proportional to $r^{n-1}$ for large $r$. On the other hand, the sets $B_{r} \cap\{u= \pm 1\}$ are thick and have Lebesgue measure proportional to $r^{n}$.

Lemma 6.1 Let $u \in \mathcal{M}_{\Omega}$. Then, for fixed $r_{1}>0$, there exist constants $r_{0}$ and $C$ depending only on the structural constants and $r_{1}$ such that

$$
\mathcal{J}_{B_{r}}(u) \leqslant C r^{n-1}
$$

for any $r \geqslant r_{0}$ provided $B_{r+r_{1}} \subset \Omega$.

Proof. Let $h$ be a smooth function so that $\left.h\right|_{B_{r-1}}=-1$ and $\left.h\right|_{\partial B_{r}}=1$. Let $u^{*}=\min \{u, h\}$. Then

$$
\begin{aligned}
\mathcal{J}_{B_{r}}(u) & \leqslant \mathcal{J}_{B_{r}}\left(u^{*}\right) \leqslant C \int_{B_{r}-B_{r-1}}\left(|\nabla u|^{p}+|\nabla h|^{p}+r^{n-1}\right) \\
& \leqslant C \int_{B_{r}-B_{r-1}}\left(|\nabla u|^{p}+r^{n-1}\right) .
\end{aligned}
$$

Let us cover $B_{r}-B_{r-1}$ with balls $\mathcal{B}_{1}, \ldots, \mathcal{B}_{N}$ of radius $1+r_{1} / 2$ with $N \leqslant C r^{n-1}$. Then, by a Caccioppoli type inequality (see [HKM93]), we infer that

$$
\int_{B_{r}-B_{r-1}}|\nabla u|^{p} \leqslant \sum_{i=1}^{N} \int_{\mathcal{B}_{i}}|\nabla u|^{p} \leqslant C \sum_{i=1}^{N} \int_{2 \mathcal{B}_{i}}|u|^{p} \leqslant C r^{n-1} .
$$

Lemma 6.2 Let $u \in \mathcal{M}_{\Omega}$. Fix $\theta_{0} \in[0,1)$ and let $\theta \in\left[-\theta_{0}, \theta_{0}\right]$. Assume that

$$
\mathcal{L}^{n}\left(\{u>\theta\} \cap B_{\rho}\right) \geqslant \mu_{0}>0 .
$$

Then, for fixed $r_{1}>0$, there exist positive constants $c$ and $r_{0}$ depending only on $r_{1}, \theta_{0}, \rho$ and $\mu_{0}$ and the structural constants such that

$$
\mathcal{L}^{n}\left(\{u>\theta\} \cap B_{r}\right) \geqslant c r^{n}
$$

for any $r \geqslant r_{0}$ provided $B_{r+r_{1}} \subset \Omega$.

An analogous statement holds for the sublevel set $\{u<\theta\}$.

Proof. Let $h$ be a smooth function so that $\left.h\right|_{B_{r-1}}=-1$ and $\left.h\right|_{\partial B_{r}}=1$. Let $u^{*}=\min \{u, h\}$ and $\beta=\min \left\{u-u^{*}, 1+\theta\right\}$. Define also

$$
\mathcal{V}(r)=\mathcal{L}^{n}\left(\{u>\theta\} \cap B_{r}\right), \quad \mathcal{A}(r)=\mathcal{L}^{n}\left(\{-1<u \leqslant \theta\} \cap B_{r}\right) .
$$


We would like to prove that

$$
[\mathcal{V}(r-1)]^{(n-1) / n}+\mathcal{A}(r-1) \leqslant C(\mathcal{V}(r)-\mathcal{V}(r-1)+\mathcal{A}(r)-\mathcal{A}(r-1)) .
$$

Indeed, from 6.1) and Lemma A.2.

$$
\mathcal{V}(\rho+k)+\mathcal{A}(\rho+k) \geqslant c(k+1)^{n}
$$

for any natural number $k$. This and Lemma 6.1 would imply Lemma 6.2

Let us now address the proof of (6.1). First of all, by definition of $\beta$,

$$
\int_{B_{r}}|\beta|^{2 n /(n-1)} \geqslant \int_{B_{r-1} \cap\{u>\theta\}}|\beta|^{2 n /(n-1)}=(1+\theta)^{2 n /(n-1)} \mathcal{L}^{n}\left(B_{r-1} \cap\{u>\theta\}\right) .
$$

Therefore, by the Sobolev inequality applied to $\beta^{2}$ and then by the Young inequality with $1 / p+$ $1 / q=1$ we obtain

$$
\begin{aligned}
{[\mathcal{V}(r-1)]^{(n-1) / n} } & \leqslant C \int_{B_{r} \cap\left\{u-u^{*} \leqslant 1+\theta\right\}}|\beta||\nabla \beta| \\
& \leqslant C\left(K \int_{B_{r} \cap\left\{u-u^{*} \leqslant 1+\theta\right\}}\left|\nabla\left(u-u^{*}\right)\right|^{p}+\frac{1}{K} \int_{B_{r} \cap\left\{u-u^{*} \leqslant 1+\theta\right\}}\left|u-u^{*}\right|^{q}\right) .
\end{aligned}
$$

Here $K>0$ is a free parameter that will be conveniently chosen in what follows. By our hypotheses on the functional and the minimality property of $u$, we deduce from the above estimate that

$$
\begin{aligned}
{[\mathcal{V}(r-1)]^{(n-1) / n} \leqslant } & C\left(K \int_{B_{r}} A\left(x, \nabla\left(u-u^{*}\right)\right)+\frac{1}{K} \int_{B_{r} \cap\left\{u-u^{*} \leqslant 1+\theta\right\}}\left|u-u^{*}\right|^{q}\right) \\
\leqslant & C\left(K \int_{\left(B_{r}-B_{r-1}\right) \cap\{u \neq-1\}}\left[A\left(x, \nabla\left(u-u^{*}\right)\right)-A(x, \nabla u)+A\left(x, \nabla u^{*}\right)\right]\right. \\
& \left.+\int_{B_{r}} Q(x)\left[\chi_{(-1,1)}\left(u^{*}\right)-\chi_{(-1,1)}(u)\right]+\frac{1}{K} \int_{B_{r} \cap\left\{u-u^{*} \leqslant 1+\theta\right\}}\left|u-u^{*}\right|^{q}\right) .
\end{aligned}
$$

By our assumptions on the functional and the gradient estimates in Theorem 4.2 , we have

$$
\int_{\left(B_{r}-B_{r-1}\right) \cap\{u \neq-1\}}\left[A\left(x, \nabla\left(u-u^{*}\right)\right)+A\left(x, \nabla u^{*}\right)\right] \leqslant C \mathcal{L}^{n}\left(\left(B_{r}-B_{r-1}\right) \cap\{u \neq-1\}\right) .
$$

Thus

$$
\begin{aligned}
{[\mathcal{V}(r-1)]^{(n-1) / n} \leqslant } & C\left(K \int_{B_{r}} Q(x)\left[\chi_{(-1,1)}\left(u^{*}\right)-\chi_{(-1,1)}(u)\right]+\frac{1}{K} \int_{B_{r} \cap\left\{u-u^{*} \leqslant 1+\theta\right\}}\left|u-u^{*}\right|^{q}\right) \\
& +C \mathcal{L}^{n}\left(\left(B_{r}-B_{r-1}\right) \cap\{u \neq-1\}\right) .
\end{aligned}
$$

To estimate the remaining two terms above, we split the domain of integration. Namely, in $B_{r-1}$, we have $u^{*}=-1$ and therefore $\chi_{(-1,1)}\left(u^{*}\right)=0$. Thus, in $B_{r-1}$ we are left with

$$
-K \int_{B_{r-1}} Q(x) \chi_{(-1,1)}(u)+\frac{1}{K} \int_{B_{r-1} \cap\{-1<u \leqslant \theta\}}|u+1|^{q} \leqslant-\mathcal{A}(r-1),
$$


by choosing $K$ suitably large. On the other hand, in $B_{r}-B_{r-1}$, we are left with the term

$$
\begin{aligned}
C \int_{\left(B_{r}-B_{r-1}\right) \cap\{u \neq-1\}}\left(Q(x)\left[\chi_{(-1,1)}\left(u^{*}\right)-\chi_{(-1,1)}(u)\right]+\right. & \left.\left|u-u^{*}\right|^{q}\right) \\
& \leqslant C \mathcal{L}^{n}\left(\left(B_{r}-B_{r-1}\right) \cap\{u \neq-1\}\right) .
\end{aligned}
$$

From these estimates, 6.1) readily follows.

Lemma 6.3 Let $u \in \mathcal{M}_{\Omega}$. Assume that $x \in\{-1<u<1\}$. Then, for fixed $r_{1}>0$, there exist positive $r_{0}$ depending only on the structural constants and $r_{1}$ such that $u$ takes both the values 1 and -1 in $B_{r_{0}}(x)$, provided $B_{r_{0}+r_{1}}(x) \subset \Omega$.

Proof. By Lemma 6.1, $u$ takes either the value -1 or +1 in $B_{r_{0} / 2}(x)$; say it takes the value -1 . If $u$ does not assume the value 1 in $B_{r_{0}}(x)$, using the linear growth from the free boundary (see Lemma 5.2], we have

$$
2 \geqslant \sup _{B_{r_{0}}(x)}(u+1) \geqslant c r_{0} \geqslant 3
$$

by choosing $r_{0}$ large enough, which is a contradiction.

We are now in a position to improve the previous density estimates:

Lemma 6.4 Let $u \in \mathcal{M}_{\Omega}$. Assume that $x \in\{-1<u<1\}$. Then, for fixed $r_{1}>0$, there exist positive $r_{0}$ and $c$ depending only on the structural constants and $r_{1}$ such that

$$
\mathcal{J}_{B_{r}(x)}(u) \geqslant c r^{n-1}
$$

for any $r \geqslant r_{0}$ provided $B_{r+r_{1}}(x) \subset \Omega$.

Proof. By Lemma 6.3 the uniform continuity of $u$ and Lemma 6.1, we have

$$
\mathcal{L}^{n}\left(B_{r}(x) \cap\{u>9 / 10\}\right) \geqslant c r^{n}, \quad \mathcal{L}^{n}\left(B_{r}(x) \cap\{u<-9 / 10\}\right) \geqslant c r^{n} .
$$

Let $a$ be the average of $u$ in $B_{r}(x)$. If $a \leqslant 0$, then

$$
\int_{B_{r}(x)}|u-a| \geqslant \int_{B_{r}(x) \cap\{u>9 / 10\}}(u-a) \geqslant \frac{9}{10} \mathcal{L}^{n}\left(B_{r}(x) \cap\{u>9 / 10\}\right) \geqslant c r^{n} .
$$

Analogously, if $a \geqslant 0$, then

$$
\int_{B_{r}(x)}|u-a| \geqslant \frac{9}{10} \mathcal{L}^{n}\left(B_{r}(x) \cap\{u<-9 / 10\}\right) \geqslant c r^{n} .
$$

So, in any case, the Poincaré and Young inequalities yield

$$
c r^{n-1} \leqslant \frac{1}{r} \int_{B_{r}(x)}|u-a| \leqslant C \int_{B_{r}(x)}|\nabla u| \leqslant C \mathcal{J}_{B_{r}(x)}(u) .
$$

Combining the density estimates above, we immediately obtain the proof of Theorem 2.2 . Besides, these estimates also imply Theorem 2.3. Indeed, in the notation of Theorem 2.3. if one 
assumes the claim to be false, there would exist $\delta>0$ and an infinitesimal sequence $\varepsilon_{n}$ for which, say, $B_{\delta}\left(x_{n}\right) \subset E$ for suitable $x_{n} \in\left\{-1<u_{\varepsilon_{n}}<1\right\}$. But then, considering $w_{\varepsilon}(x):=u_{\varepsilon}(\varepsilon x)$ (see the end of Section 3 for the rescaling argument) and applying the density estimates we would get, after scaling back to $u_{\varepsilon}$,

$$
\mathcal{L}^{n}\left(B_{\delta / 2}\left(x_{n}\right) \cap\left\{u_{\varepsilon_{n}}=-1\right\}\right) \geqslant c \delta^{n}
$$

for some $c>0$. But then

$$
\int_{B_{\delta / 2}\left(x_{n}\right)}\left|u_{\varepsilon_{n}}-u_{0}\right| \geqslant 2 c \delta^{n},
$$

contradicting the hypothesis. This proves Theorem 2.3 .

\section{Geometric Lemma}

We show in this section that for a minimizer $u$ of $\mathcal{J}_{\Omega}$ the sets $B \cap\{u= \pm 1\}$, where $B$ is a large ball centered in the jet $\{-1<u<1\}$, contain balls proportional to $B$. This is a consequence of the density estimates in Theorem 2.2 and the following result, known as the Geometric Lemma.

Lemma 7.1 (Geometric Lemma) Let $\beta \subset E \subset \mathbb{R}^{n}$, and let $\mu$ and $v$ be Radon measures on $\mathbb{R}^{n}$. Take $x_{0} \in \beta$. Fix $r>0$. Assume that there exists $\delta^{\prime} \in(0,1 / 10]$ such that:

(i) for all $x \in B_{r}\left(x_{0}\right)$ and $\varrho \in\left[\delta^{\prime} r, r / 10\right]$,

$$
\mu\left(B_{\varrho}(x)\right) \leqslant C \varrho^{n} ;
$$

(ii) for all $x \in \beta \cap B_{r}\left(x_{0}\right)$ and $\varrho \in\left[\delta^{\prime} r, r / 10\right]$

$$
v\left(B_{\varrho}(x)\right) \geqslant c \varrho^{n-1} ;
$$

(iii) $\mu\left(E \cap B_{r}\left(x_{0}\right)\right) \geqslant c r^{n}$;

(iv) $v\left(B_{2 r}\left(x_{0}\right)\right) \leqslant C r^{n-1}$.

Then there exists $\delta$, depending only on $n$ and on the constants involved in (i)-(iv) above, so that, if $\delta^{\prime} \leqslant \delta$, we can find $\bar{x} \in B_{r}\left(x_{0}\right)$ such that

$$
B_{\delta r}(\bar{x}) \subset(E-\beta) \cap B_{2 r}\left(x_{0}\right) .
$$

Proof. For a complete proof see [Val01, Lemma 6.1]. Here we just outline the rough idea of the proof. We first cover $\beta$ with balls of radius $\delta r$; since $\beta$ behaves like an $(n-1)$-dimensional set, we need $O\left(1 / \delta^{n-1}\right)$ such balls to cover $\beta$. We then extend this cover to the whole set $E$; since $E$ behaves like an $n$-dimensional set, we need $O\left(1 / \delta^{n}\right)$ such balls to cover $E$. Therefore, if $\delta$ is small enough, there is at least one ball in the cover of $E$ that does not touch $\delta$.

Here is an application of the Geometric Lemma to our case:

Proposition 7.2 Let $u \in \mathcal{M}_{\Omega}$. Then, for any fixed $r_{1}>0$, there exist positive constants $\kappa$ and $r_{0}$, depending only on the structural constants and $r_{1}$, such that for any $x \in\{-1<u<1\}$ and any $r \geqslant r_{0}$, there exist $z_{1}$ and $z_{2}$ with

$$
B_{\kappa r}\left(z_{1}\right) \subset\{u=1\} \cap B_{r}(x) \text { and } \quad B_{\kappa r}\left(z_{2}\right) \subset\{u=-1\} \cap B_{r}(x),
$$

provided $B_{2 r+r_{1}}(x) \subset \Omega$.

This follows easily by applying the Geometric Lemma to the sets $E:=\{u>-1\}$ or $E:=$ $\{u<1\}$, and $\beta:=\{|u|<1\}$, with $\mu:=\mathcal{L}^{n}$ and $\nu(B):=\mathcal{J}_{B}(u)$. Notice that the hypotheses of the Geometric Lemma are satisfied in view of the density estimates (see Theorem 2.2). 


\section{Plane-like minimizers}

We now prove Theorem 2.4. We consider $\omega \in \mathbb{Q}^{n}-\{0\}$ (the case $\omega \in \mathbb{R}^{n}-\mathbb{Q}^{n}$ then follows by uniform approximation). We denote by $\mathcal{M}_{M}^{\omega}$ the set of all periodic constrained minimizers in the strip

$$
S_{M}^{\omega}:=\left\{x \in \mathbb{R}^{n}: x \cdot \omega \in[0, M|\omega|]\right\} .
$$

When $\omega \in \mathbb{Q}^{n}$, we can recover compactness by making some identifications in the space. Namely, we consider the following relation $\sim$ induced by $\omega$ :

$$
x \sim y \quad \text { iff } \quad x-y=k \in \mathbb{Z}^{n} \text { with } k \cdot \omega=0 .
$$

We denote by $\tilde{S}_{M}^{\omega}=S_{M}^{\omega} / \sim$ the quotient space of this relation (which is topologically equivalent to the product of an $(n-1)$-dimensional torus and a real interval).

In what follows we consider the functional $\mathcal{J}$ on functions $u$ periodic with respect to the identification $\sim$ in $S_{M}^{\omega}$, and we let $\mathcal{J}_{\tilde{S}_{M}^{\omega}}$ be the integral over a fundamental region with respect to the relation $\sim$.

We also set

$$
\begin{aligned}
\mathcal{X}_{M}^{\omega}:=\left\{u \in L_{\mathrm{loc}}^{1}\left(\tilde{S}_{M}^{\omega}\right): \nabla u\right. & \in L^{p}\left(\tilde{S}_{M}^{\omega}\right), \\
& \quad u(x)=1 \text { on } x \cdot \omega=0 \text { and } u(x)=-1 \text { on } x \cdot \omega=M|\omega|\} .
\end{aligned}
$$

In what follows, we assume $M \geqslant 10$, in order to avoid degeneracy caused by "too thin" strips.

First of all, we notice the following elementary fact: for any $u, v \in L_{\text {loc }}^{1}\left(\tilde{S}_{M}^{\omega}\right)$ with $\nabla u, \nabla v \in$ $L^{2}\left(\tilde{S}_{M}^{\omega}\right)$, one has

$$
\mathcal{J}_{S_{M}^{\omega}}(u)+\mathcal{J}_{S_{M}^{\omega}}(v)=\mathcal{J}_{S_{M}^{\omega}}(\min \{u, v\})+\mathcal{J}_{S_{M}^{\omega}}(\max \{u, v\}) .
$$

Therefore, if $u, v \in \mathcal{M}_{M}^{\omega}$, then $\min \{u, v\}, \max \{u, v\} \in \mathcal{M}_{M}^{\omega}$.

Now, we prove a geometric property concerning the global behavior of the minimizers. If $k \in \mathbb{R}^{n}$, we denote by $\mathcal{T}_{k}$ the translation by the vector $k$. Also, we set $\mathcal{T}_{k} u(x):=u(x-k)$.

DEFINITION 8.1 Let $E \subset \mathbb{R}^{n}$ and $\varpi \in \mathbb{R}^{n}$. We say that $E$ has the Birkhoff property with respect to the vector $\varpi$ if:

- for any $k \in \mathbb{Z}^{n}$ so that $k \cdot \varpi \leqslant 0, \mathcal{I}_{k} E \subset E$;

- for any $k \in \mathbb{Z}^{n}$ so that $k \cdot \varpi \geqslant 0, \mathcal{T}_{k} E \supset E$.

It follows from the above definition that if $E \subset \mathbb{R}^{n}$ satisfies the Birkhoff property with respect to $\omega$, then there exists a constant $\varrho$, depending only on $n$, such that if $\mathcal{C}(E)$ contains a ball of radius $\varrho$, then $\mathcal{C}(E)$ contains a strip of width 1 that intersects the ball.

It is convenient to introduce the notion of minimal minimizer. Following [CdlL01] and [Val01], we define

$$
u_{M}^{\omega}(x):=\min _{u \in \mathcal{M}_{M}^{\omega}} u(x)
$$

By a standard compactness argument, the reader may convince himself that the definition is legitimate. Let us now show that the level sets of the minimal minimizer have the Birkhoff property.

Proposition 8.2 Let $\vartheta \in \mathbb{R}$. Then the set $\left\{u_{M}^{\omega}>\vartheta\right\}$ has the Birkhoff property with respect to $\omega$. Namely:

- if $k \in \mathbb{Z}^{n}$ and $k \cdot \omega \leqslant 0$, then $\left\{u_{M}^{\omega}>\vartheta\right\} \supset\left\{\mathcal{T}_{k} u_{M}^{\omega}>\vartheta\right\}$;

- if $k \in \mathbb{Z}^{n}$ and $k \cdot \omega \geqslant 0$, then $\left\{u_{M}^{\omega}>\vartheta\right\} \subset\left\{\mathcal{T}_{k} u_{M}^{\omega}>\vartheta\right\}$. 
This follows easily from 8.3 and the definition of minimal minimizer, by looking at $\min \left\{u_{M}^{\omega}, \mathcal{T}_{k} u_{M}^{\omega}\right\}$ and $\max \left\{u_{M}^{\omega}, \mathcal{T}_{k} u_{M}^{\omega}\right\}$.

We now show that the minimal minimizer enjoys the doubling property (also known in the literature as no-symmetry-breaking property). It states that the minimal minimizer defined in a set whose period is a multiple of the original one still has the original period. In some sense, the doubling property forces the minimal minimizers to exhibit the same periodicity as the one induced by the functional. More precisely:

PROPOSITION 8.3 Let $v_{1}, \ldots, v_{n-1} \in \mathbb{N}-\{0\}$. Let $\sim$ be the equivalence relation defined in 8.1]. Assume that the $(n-1)$-dimensional lattice induced by $\sim$ is spanned by $k_{1}^{*}, \ldots, k_{n-1}^{*} \in \mathbb{Z}^{n}$, i.e. any $k \in \mathbb{Z}^{n}$ such that $\omega \cdot k=0$ can be written as

$$
k=\sum_{i=1}^{n-1} m_{i} k_{i}^{*}
$$

for suitable $m_{1}, \ldots, m_{n-1} \in \mathbb{Z}$. Consider the equivalence relation

$$
x \simeq y \quad \text { iff } \quad x-y=\sum_{i=1}^{n-1} m_{i} v_{i} k_{i}^{*}
$$

for suitable $m_{1}, \ldots, m_{n-1} \in \mathbb{Z}$. Let $u$ and $v$ be the minimal minimizers in $S_{M}^{\omega} / \sim$ and $S_{M}^{\omega} / \simeq$, respectively (constrained to be 1 when $\omega \cdot x=0$, and -1 when $\omega \cdot x=M|\omega|$ ). Then $v$ is also periodic with respect to $\sim$, i.e.

$$
v(x+k)=v(x)
$$

for any $k \in \mathbb{Z}^{n}$ such that $\omega \cdot k=0$. Also,

$$
v=u
$$

Proof. Since (8.4) and the definition of minimal minimizer directly imply 8.5 , we just consider the proof of (8.4). The periodicity induced by $\simeq$ on $v$ reads

$$
v\left(x+\sum_{i=1}^{n-1} m_{i} v_{i} k_{i}^{*}\right)=v(x)
$$

for any $m_{1}, \ldots, m_{n-1} \in \mathbb{Z}$ and any $x \in \mathbb{R}^{n}$, or equivalently,

$$
v\left(x+m_{i} v_{i} k_{i}^{*}\right)=v(x)
$$

for any $m_{i} \in \mathbb{Z}$ and any $x \in \mathbb{R}^{n}$. We want to prove that

$$
v\left(x+\sum_{i=1}^{n-1} m_{i} k_{i}^{*}\right)=v(x)
$$

for any $m_{1}, \ldots, m_{n-1} \in \mathbb{Z}$ and any $x \in \mathbb{R}^{n}$, or equivalently, that

$$
v\left(x+k_{i}^{*}\right)=v(x)
$$


for any $x \in \mathbb{R}^{n}$. Let us do the case $i=1$, the others being analogous. Let

$$
\begin{aligned}
v_{*}(x):= & \min \left\{v(x), v\left(x+k_{1}^{*}\right), v\left(x+2 k_{1}^{*}\right), \ldots, v\left(x+\left(v_{1}-1\right) k_{1}^{*}\right)\right\} \\
= & \min \left\{v(x), \min \left\{v\left(x+k_{1}^{*}\right), \min \left\{v\left(x+k_{2}^{*}\right), \ldots\right.\right.\right. \\
& \left.\left.\ldots, \min \left\{v\left(x+\left(v_{1}-2\right) k_{1}^{*}\right), v\left(x+\left(v_{1}-1\right) k_{1}^{*}\right)\right\}\right\}\right\} .
\end{aligned}
$$

Since $v_{*}$ satisfies the same constraints and periodicity as $v$, it is also a minimizer, thanks to 8.3 . From the fact that $v$ is the minimal minimizer we thus infer $v \leqslant v_{*}$; hence, as $v_{*} \leqslant v$ by construction, 8.6 is proved.

This doubling property will play a role in Proposition 8.5 below.

We now show that constrained minimizers are indeed unconstrained and class $A$, provided that the strip is wide enough. In order to clearly state the next result, we introduce the following notation. Let $m \leqslant M$ and define

$$
S_{m, M}^{\omega}:=\left\{x \in \mathbb{R}^{n}: x \cdot \omega \in[m|\omega|, M|\omega|]\right\}
$$

Set also

$$
\begin{aligned}
\mathcal{X}_{m, M}^{\omega}:=\left\{u \in L_{\mathrm{loc}}^{1}\left(\tilde{S}_{m, M}^{\omega}\right): \nabla u \in L^{p}\left(\tilde{S}_{m, M}^{\omega}\right),\right. \\
\\
\quad u(x)=1 \text { on } x \cdot \omega=m|\omega| \text { and } u(x)=-1 \text { on } x \cdot \omega=M|\omega|\} .
\end{aligned}
$$

We also denote by $\mathcal{M}_{m, M}^{\omega}$ the set of all periodic constrained minimizers in the strip $S_{m, M}^{\omega}$. Of course, $S_{M}^{\omega}=S_{0, M}^{\omega}, \mathcal{X}_{M}^{\omega}=\mathcal{X}_{0, M}^{\omega}$ and $\mathcal{M}_{M}^{\omega}=\mathcal{M}_{0, M}^{\omega}$. Also, we define the minimal minimizer

$$
u_{m, M}^{\omega}(x):=\min _{u \in \mathcal{M}_{m, M}^{\omega}} u(x)
$$

To simplify the notation, we also set $u_{M}^{\omega}:=u_{0, M}^{\omega}$.

We now prove that the minimal minimizer constrained in a strip wide enough is indeed unconstrained.

Proposition 8.4 There exists $M_{0}>0$, depending only on the structural constants, such that if $M \geqslant M_{0}$, then $u_{M}^{\omega}=u_{-a, M+b}^{\omega}$ for any $a, b \geqslant 0$.

Proof. Let $u=u_{-a, M+b}^{\omega}$. Let $S$ be a strip of width 3 around the hyperplane $L:=\{x \cdot \omega=M / 2\}$. We now prove that there exists a strip $H$ of width 1 parallel to the constraints and at distance from $L$ universally bounded, on which

$$
\text { either } u=-1 \quad \text { or } \quad u=1 \text {. }
$$

For that, we may assume that there exists a point $x^{*} \in S$ so that $u\left(x^{*}\right) \in(-1,1)$, otherwise 8.7 is obviously true. Then from Proposition 7.2 there exists a ball (whose radius is large if $M$ is large), contained in $\{u=-1\}=\mathcal{C}\{u>-1\}$. Hence, we obtain a strip of width 1 intersecting the ball and contained in $\{u=-1\}$. This finishes the proof of 8.7 .

Since $u$ is the minimal minimizer, it cannot be that $u=1$ in $S$, otherwise we could lower it. Hence $u=-1$ in $S$ and thus, for any $a, b \geqslant 0$,

$$
u_{-a, M+b}^{\omega} \in \mathcal{M}_{-a, M}^{\omega} .
$$


Roughly speaking, this says that "the upper constraint has become irrelevant". We now show that the lower constraint is not necessary either. Indeed, given any $v \in \mathcal{X}_{-a, M+b}^{\omega}$, we can translate it up by an integer vector to a $w \in \mathcal{X}_{0, M+\hat{b}}^{\omega}$ for a suitable $\hat{b} \geqslant b$. By 8.8 , the energy of $w$ (which agrees with the one of $v$ and is not less than that of $u_{M+\hat{b}}^{\omega}$ ) is not less than that of $u_{0, M}^{\omega}$. Therefore a minimizer in $\mathcal{X}_{0, M}^{\omega}$ a minimizer in $\mathcal{X}_{-a, M+b}^{\omega}$.

We now show that the minimal minimizer in a wide enough strip is indeed a class $A$ minimizer.

Proposition 8.5 There exists a universal constant $M_{0}$ so that if $M \geqslant M_{0}$, then $u_{M}^{\omega}$ is a class $A$ minimizer.

Proof. Given a compact perturbation, by the doubling property (see Proposition 8.3 , we can always consider $u_{M}^{\omega}$ to be periodic with a period larger than the diameter of the perturbation and (in view of Proposition 8.4 as a minimizer in a strip of width greater than the size of the perturbation.

This completes the proof of (T1) in Theorem 2.4 indeed, the case of irrational frequency can be easily inferred from the rational frequency case by a limiting argument, by noticing that $M_{0}$ does not depend on the frequency.

We now prove (T2) of Theorem 2.4 We will keep on assuming that $\omega \in \mathbb{Q}^{n}-\{0\}$.

PROPOSITION 8.6 There exists a universal constant $M_{0}$ so that if $M \geqslant M_{0}$, then $\mathcal{M}_{M}^{\omega} \subset$ $\mathcal{M}_{-a, M+b}^{\omega}$ for any $a, b \geqslant 0$.

Proof. Take $u \in \mathcal{M}_{M}^{\omega}$ and $v \in \mathcal{X}_{-a, M+b}^{\omega}$ and consider the minimal minimizer $u_{M}^{\omega}$ in $\tilde{S}_{M}^{\omega}$. Since $u, u_{M}^{\omega} \in \mathcal{M}_{M}^{\omega}$,

$$
\mathcal{J}_{\tilde{S}_{M}^{\omega}}\left(u_{M}^{\omega}\right)=\mathcal{J}_{\tilde{S}_{M}^{\omega}}(u) .
$$

Since $|u|=1=\left|u_{M}^{\omega}\right|$ when $x \cdot \omega \leqslant 0$ or $x \cdot \omega \geqslant M$,

$$
\mathcal{J}_{\tilde{S}_{M}^{\omega}}(u)=\mathcal{J}_{\tilde{S}_{-a, M+b}^{\omega}}(u) \quad \text { and } \quad \mathcal{J}_{\tilde{S}_{M}^{\omega}}\left(u_{M}^{\omega}\right)=\mathcal{J}_{\tilde{S}_{-a, M+b}^{\omega}}\left(u_{M}^{\omega}\right) .
$$

Also, by Proposition $8.4 u_{M}^{\omega}$ still minimizes $\mathcal{J}$ in $\tilde{S}_{-a, M+b}^{\omega}$, and so

$$
\mathcal{J}_{\tilde{S}_{-a, M+b}^{\omega}}(v) \geqslant \mathcal{J}_{\tilde{S}_{-a, M+b}^{\omega}}\left(u_{M}^{\omega}\right) .
$$

Collecting the relations above yields $\mathcal{J}_{\tilde{S}_{-a, M+b}^{\omega}}(v) \geqslant \mathcal{J}_{\tilde{S}_{-a, M+b}^{\omega}}(u)$.

Proposition 8.7 There exists a universal constant $M_{0}$ so that if $M \geqslant M_{0}$, then any constrained minimizer $u \in \mathcal{M}_{M}^{\omega}$ is a class $A$ minimizer.

Proof. Let $B \subset \mathbb{R}^{n}$ be a ball and take $\varphi \in C_{0}^{\infty}(B)$. Let $S$ be a strip obtained from $\tilde{S}_{M}^{\omega}$ by enlarging its width and by repeating its periodicity $v$ times in such a way that it well contains the ball $B$ (of course, if we repeat the periodicity $v_{i}$ times in the $i$ th direction, we have $v=v_{1} \cdots v_{n-1} \in \mathbb{N}-\{0\}$ ). Hence, if $v$ enjoys the periodicity induced by $\sim$, i.e. if $v(x)=v(x+k)$ whenever $k \cdot \omega=0$, we have

$$
\mathcal{J}_{S}(v)=v \mathcal{J}_{\tilde{S}_{M}^{\omega}}(v)
$$

Let now $u \in \mathcal{M}_{M}^{\omega}$ and consider the minimal minimizer $u_{M}^{\omega}$. By the doubling property (see Proposition 8.3 and Proposition 8.4, $u_{M}^{\omega}$ still minimizes $\mathcal{J}$ in $S$, whence

$$
\mathcal{J}_{S}\left(u_{M}^{\omega}\right) \leqslant \mathcal{J}_{S}(u+\varphi) \text {. }
$$


Also, since both $u$ and $u_{M}^{\omega}$ are minimizers in $\tilde{S}_{M}^{\omega}$,

$$
\mathcal{J}_{\tilde{S}_{M}^{\omega}}(u)=\mathcal{J}_{\tilde{S}_{M}^{\omega}}\left(u_{M}^{\omega}\right) .
$$

Moreover, $u=u+\varphi$ in $S-B$. Therefore, by the relations above,

$$
\begin{aligned}
\mathcal{J}_{B}(u+\varphi)-\mathcal{J}_{B}(u) & =\mathcal{J}_{S}(u+\varphi)-\mathcal{J}_{S}(u) \\
& \geqslant \mathcal{J}_{S}\left(u_{M}^{\omega}\right)-\mathcal{J}_{S}(u)=v \mathcal{J}_{\tilde{S}_{M}^{\omega}}\left(u_{M}^{\omega}\right)-v \mathcal{J}_{\tilde{S}_{M}^{\omega}}(u)=0 .
\end{aligned}
$$

Thus, (T2) in Theorem 2.4 follows from Propositions 8.6 and 8.7 This completes the proof of Theorem 2.4

\section{A. Appendix}

In the proof of Lemma 4.2 we have used an approximation of minimizers of $\mathcal{F}$ with solutions of the homogeneous equation $\operatorname{div} a(x, \nabla u)=0$. Namely, let

$$
\mathcal{F}_{\Omega}(w)=\int_{\Omega}\left[A(x, \nabla w)+Q(x) \chi_{(0, \infty)}(w)\right],
$$

where $A(x, \eta)$ and $a(x, \eta)=D_{\eta} A(x, \eta)$ satisfy (H1)-(H6) and

$$
0 \leqslant Q(x) \leqslant \varepsilon .
$$

A consequence of conditions (H1)-(H3) is the following useful inequality:

$$
\left(a(x, \eta)-a\left(x, \eta^{\prime}\right)\right) \cdot\left(\eta-\eta^{\prime}\right) \geqslant \lambda \begin{cases}\left|\eta-\eta^{\prime}\right|^{2}\left(|\eta|+\left|\eta^{\prime}\right|\right)^{p-2}, & 1<p \leqslant 2, \\ \left|\eta-\eta^{\prime}\right|^{p} & p \geqslant 2,\end{cases}
$$

(see, e.g., [Tol84]).

Lemma A.1 Let $w \in W^{1, p}\left(B_{1}\right)$ be an absolute minimizer of $\mathcal{F}_{B_{1}}$. Let also $w_{h} \in W^{1, p}\left(B_{3 / 4}\right)$ satisfy

$$
\operatorname{div} a\left(x, \nabla w_{h}\right)=0, \quad w_{h}-w \in W_{0}^{1, p}\left(B_{3 / 4}\right) .
$$

Then

$$
\left\|w-w_{h}\right\|_{W^{1, p}\left(B_{3 / 4}\right)} \leqslant C \varepsilon^{\alpha / p}\|w\|_{L^{p}\left(B_{1}\right)}^{1-\alpha}
$$

with $\alpha=\min (1, p / 2)$, where $C_{0}$ depends only on the structural constants of $A$.

Proof. From the minimality of $w$ in $B=B_{3 / 4}$ we have

$$
\int_{B}\left[A(x, \nabla w)+Q(x) \chi_{(0, \infty)}(w)\right] \leqslant \int_{B}\left[A\left(x, \nabla w_{h}\right)+Q(x)\right],
$$

or

$$
\int_{B}\left[A(x, \nabla w)-A\left(x, \nabla w_{h}\right)\right] \leqslant \varepsilon \int_{B} \chi_{\{u=0\}} .
$$

Set now

$$
w^{s}(x)=s w(x)+(1-s) w_{h}(x), \quad 0 \leqslant s \leqslant 1 .
$$


Clearly $w^{0}=w_{h}$ and $w^{1}=w$. We thus obtain

$$
\begin{aligned}
\int_{B}\left[A(x, \nabla w)-A\left(x, \nabla w_{h}\right)\right] & =\int_{0}^{1} \mathrm{~d} s \int_{B} a\left(x, \nabla w^{s}\right) \cdot \nabla\left(w-w_{h}\right) \\
& =\int_{0}^{1} \mathrm{~d} s \int_{B}\left(a\left(x, \nabla w^{s}\right)-a\left(x, \nabla w_{h}\right)\right) \cdot \nabla\left(w-w_{h}\right) \\
& =\int_{0}^{1} \frac{\mathrm{d} s}{s} \int_{B}\left(a\left(x, \nabla w^{s}\right)-a\left(x, \nabla w_{h}\right)\right) \cdot \nabla\left(w^{s}-w_{h}\right),
\end{aligned}
$$

where in the second step we used the fact that $\int_{B} a\left(x, \nabla w_{h}\right) \cdot \nabla\left(w-w_{h}\right)=0$.

Next, we apply the inequality (A.1). For $p \geqslant 2$ we obtain

$$
\begin{aligned}
\int_{B}\left[A(x, \nabla w)-A\left(x, \nabla w_{h}\right)\right] & \geqslant \lambda \int_{0}^{1} \frac{\mathrm{d} s}{s} \int_{B}\left|\nabla\left(w^{s}-w_{h}\right)\right|^{p} \\
& =\lambda \int_{0}^{1} s^{p-1} \mathrm{~d} s \int_{B}\left|\nabla\left(w-w_{h}\right)\right|^{p}
\end{aligned}
$$

and consequently

$$
\int_{B}\left|\nabla\left(w-w_{h}\right)\right|^{p} \leqslant C \varepsilon \int_{B} \chi_{\{u=0\}}
$$

In the case $1<p \leqslant 2$ we have

$$
\begin{aligned}
\int_{B}\left[A(x, \nabla w)-A\left(x, \nabla w_{h}\right)\right] & \geqslant \lambda \int_{0}^{1} \frac{\mathrm{d} s}{s} \int_{B}\left|\nabla\left(w^{s}-w_{h}\right)\right|^{2}\left(\left|\nabla w^{s}\right|+\left|\nabla w_{h}\right|\right)^{p-2} \\
& \geqslant c \int_{0}^{1} s \mathrm{~d} s \int_{B}\left|\nabla\left(w-w_{h}\right)\right|^{2}\left(|\nabla w|+\left|\nabla w_{h}\right|\right)^{p-2} \\
& \geqslant c \int_{B}\left|\nabla\left(w-w_{h}\right)\right|^{2}\left(|\nabla w|+\left|\nabla w_{h}\right|\right)^{p-2} .
\end{aligned}
$$

On the other hand, using the Hölder inequality, we have

$$
\int_{B}\left|\nabla\left(w-w_{h}\right)\right|^{p} \leqslant\left(\int_{B}\left|\nabla\left(w-w_{h}\right)\right|^{2}\left(|\nabla w|+\left|\nabla w_{h}\right|\right)^{p-2}\right)^{p / 2}\left(\int_{B}\left(|\nabla w|+\left|\nabla w_{h}\right|\right)^{p}\right)^{1-p / 2},
$$

hence

$$
\int_{B}\left|\nabla\left(w-w_{h}\right)\right|^{p} \leqslant C \varepsilon^{p / 2}\left(\int_{B} \chi_{\{w=0\}}\right)^{p / 2}\left(\int_{B_{1}} w^{p}\right)^{1-p / 2},
$$

where we have used the estimate $\int_{B}\left|\nabla w_{h}\right|^{p} \leqslant C \int_{B}|\nabla w|^{p}$ and the Caccioppoli type inequality

$$
\int_{B}|\nabla w|^{p} \leqslant C \int_{B_{1}} w^{p}
$$

(see [HKM93, Lemma 3.27]). The lemma follows now from [A.2]-[A.3].

The following estimate was used in the proof of Lemma 6.2 when applying the recurrence relationship (6.1). 
LEMMA A.2 Let $v_{k}, a_{k} \in \mathbb{R}$ satisfy $v_{k} \geqslant 0, a_{k} \geqslant 0, v_{k-1}+a_{k-1} \leqslant v_{k}+a_{k}, v_{0}+a_{0} \geqslant c_{0}>0$ and

$$
v_{k-1}^{(n-1) / n}+a_{k-1} \leqslant C\left(v_{k}-v_{k-1}+a_{k}-a_{k-1}\right)
$$

for any $k \in \mathbb{N}, k \geqslant 1$, for some positive $c_{0}$ and $C$. Then there exists a positive $c$, depending only on $C$ and $c_{0}$, such that

$$
v_{k}+a_{k} \geqslant c(k+1)^{n}
$$

for any $k \in \mathbb{N}$.

Proof. First of all, notice that, if $C_{*}$ is large enough,

$$
C_{*} x+1 \geqslant(1+x)^{n}, \quad \forall x \in[0,1] .
$$

Also, if $k \leqslant 2 C C_{*}$,

$$
v_{k}+a_{k} \geqslant v_{0}+a_{0} \geqslant c_{0} \geqslant c(k+1)^{n},
$$

provided $c \leqslant c_{0} /\left(2 C C_{*}+1\right)^{n}$. Hence we can assume $k \geqslant 2 C C_{*}$. We make an iterative argument: we assume

$$
v_{k-1}+a_{k-1} \geqslant c k^{n}
$$

and we prove $\mathrm{A} .4$.

In fact, in order to prove (A.4), by (A.5), it is enough to prove

$$
v_{k}+a_{k} \geqslant c k^{n}\left(C_{*} / k+1\right),
$$

since (A.4) then follows from (A.7) applied with $x:=1 / k$.

Hence, we now prove A.7. From the hypothesis and A.6, we have

$$
v_{k}+a_{k} \geqslant \frac{1}{C}\left(v_{k-1}^{(n-1) / n}+a_{k-1}\right)+c k^{n} .
$$

Also, using again A.6, we infer that either $v_{k-1} \geqslant c k^{n} / 2$ or $a_{k-1} \geqslant c k^{n} / 2$.

If $v_{k-1} \geqslant c k^{n} / 2$, then from A.8,

$$
v_{k}+a_{k} \geqslant c\left(C_{*} k^{n-1}+k^{n}\right),
$$

provided $C_{*} \leqslant 1 /\left(C 2^{(n-1) / n} c^{1 / n}\right)$, which holds if $c$ is small enough. This proves A.7 in this case.

On the other hand, if $a_{k-1} \geqslant c k^{n} / 2$, then from A.8 we get

$$
v_{k}+a_{k} \geqslant c k^{n}\left(\frac{1}{2 C}+1\right)
$$

which implies $(\mathrm{A} .7)$ since $k \geqslant 2 C C_{*}$.

\section{Acknowledgments}

The authors thank Henrik Shahgholian for suggesting the problems considered in this paper. EV thanks Luis Caffarelli for an invitation to Austin (on which occasion the present collaboration started) and for his support and encouragement. EV's research is partially supported by MURST Variational Methods and Nonlinear Differential Equations and GNAFA. 


\section{REFERENCES}

[AC81] Alt, H. W. \& CAFFARELli, L. A. Existence and regularity for a minimum problem with free boundary. J. Reine Angew. Math. 325 (1981), 105-144. Zbl 0449.35105 MR 0618549

[Bou90] Bouchitté, G. Singular perturbations of variational problems arising from a two-phase transition model. Appl. Math. Optim. 21 (1990), 289-314. Zbl 0695.49003 MR 1036589

[CC95] CAFFARELl, L. A. \& CóRdobA, A. Uniform convergence of a singular perturbation problem. Comm. Pure Appl. Math. 48 (1995), 1-12. Zbl 0829.49013 MR 1310848

[Cdil01] Caffarelli, L. A. \& De la Llave, R. Planelike minimizers in periodic media. Comm. Pure Appl. Math. 54 (2001), no. 12, 1403-1441. Zbl 1036.49040 MR 1852978

[Dac89] Dacorogna, B. Direct Methods in the Calculus of Variations. Appl. Math. Sci. 78, Springer, Berlin (1989). Zbl 0703.49001 MR 0990890

[DP03] DAnielli, D. \& Petrosyan, A. A minimum problem with free boundary for a degenerate quasilinear operator. Calc. Var. Partial Differential Equations, to appear.

[GG82] Giaquinta, M. \& GiUsti, E. On the regularity of the minima of variational integrals. Acta Math. 148 (1982), 31-46. Zbl 0494.49031 MR 0666107

[HKM93] Heinonen, J., KilpeläInen, T., \& Martio, O. Nonlinear Potential Theory of Degenerate Elliptic Equations, Oxford Sci. Publ., Oxford Univ. Press (1993). Z Zbl 0780.31001 MR 1207810

[To184] TOLKSDORF, P. Regularity for a more general class of quasilinear elliptic equations. $J$. Differential Equations 51 (1984), 126-150. Zbl 0488.35017 MR 0727034

[Tru67] Trudinger, N. S. On Harnack type inequalities and their application to quasilinear elliptic equations. Comm. Pure Appl. Math. 20 (1967), 721-747. Zbl 0153.42703 MR 0226198

[Val01] VAldinOcI, E. Plane-like minimizers in periodic media: jet flows and Ginzburg-Landau-type functionals. J. Reine Angew. Math. 574 (2004), 147-185. Z Zbl pre02104132 MR 2099113 